\title{
$\begin{array}{ll}\text { Research Square } & \text { Preprints are preliminary reports that have not undergone peer review. } \\ \text { They should not be considered conclusive, used to inform clinical practice, } \\ \text { or referenced by the media as validated information }\end{array}$
}

\section{Elevated Ambulatory Blood Pressure Measurements Are Associated With a Progressive Form of Fabry Disease}

\author{
Federica Rossi ( $\sim$ f.rossi92@campus.unimib.it) \\ Einar Svarstad \\ Universitetet i Bergen \\ Hassan Elsaid \\ Universitetet i Bergen \\ Agnese Binaggia \\ Azienda Socio Sanitaria Territoriale di Monza \\ Letizia Roggero \\ Universita degli Studi di Milano-Bicocca \\ Sara Auricchio \\ Azienda Socio Sanitaria Territoriale di Monza \\ Hans-Peter Marti \\ Universitetet i Bergen \\ Federico Pieruzzi \\ Universita degli Studi di Milano-Bicocca
}

Department of Medicine and Surgery, University of Milano-Bicocca, Monza, Italy, Nephrology and Dialysis Unit, ASST-Monza, San Gerardo Hospital, Monza, Italy https://orcid.org/0000-0002-1509-1190

\section{Research}

Keywords: Anderson-Fabry disease, lysosomal storage disorder, hypertension, masked hypertension, blood pressure monitoring

Posted Date: August 19th, 2020

DOI: https://doi.org/10.21203/rs.3.rs-59388/v1

License: (c) (i) This work is licensed under a Creative Commons Attribution 4.0 International License. Read Full License

Version of Record: A version of this preprint was published at High Blood Pressure \& Cardiovascular Prevention on April 12th, 2021. See the published version at https://doi.org/10.1007/s40292-021-00450-0. 


\section{Abstract}

Background and objectives

Published data on hypertension incidence and management in Anderson-Fabry disease are scanty and it remains to be shown how much an elevated blood pressure contributes to the patient's organ damage. Therefore, we have assessed blood pressure values and their correlations with clinical findings in a cohort of Fabry patients.

Methods

Between January 2015 and May 2019, all adult Fabry patients ( $n=32 ; n=24$ females, $n=8$ males) referred to our institute were enrolled. Data regarding hypertension were obtained by ambulatory blood pressure monitoring, home self-monitoring and repeated office measurements. Organ involvement and hypertension risk factors were also evaluated.

Results

The ambulatory monitoring revealed elevated blood pressure in $18.75 \%(n=6)$ of the Fabry population and $50 \%$ of this group was diagnosed with masked hypertension. All these patients were females and they presented a lower glomerular filtration rate and a more advanced cardiac hypertrophy compared with normotensive subjects. They were mostly (66.7\%) affected by a progressive form of the disease while the majority of the normotensives (84.6\%) were stable. No correlation was found between the category of GLA mutation and the development of hypertension.

Conclusion

Newly detected hypertension can be found in a restricted portion of stable Fabry patients while it becomes more prevalent among clinically progressive cases. The use of the ambulatory blood pressure monitoring is of paramount importance to reveal masked hypertension which can contribute to the progressive worsening of the organ damage. Therefore, a proper diagnosis and therapy of hypertension may improve the outcome of Fabry patients.

\section{Introduction}

Anderson-Fabry disease (AFD) is a rare X-linked sphingolipid storage disorder caused by the mutation in the GLA gene leading to a deficient activity of the lysosomal enzyme a-galactosidase A ( $a-G a l ~ A)$. The reduced or absent activity of the enzyme results in progressive lysosomal accumulation of globotriaosylceramide (Gb3) and its derivates, including globotriaosylsphingosine (lyso-Gb3), within different body cells ${ }^{1}$.

The phenotypic spectrum of the disease comprises a "classic" form characterized by a total, or almost total, absence of a-Gal A activity and an early onset of the symptoms, generally during childhood or adolescence, with neuropathic pain, skin lesions (angiokeratomas), gastrointestinal symptoms and corneal opacities (cornea verticillata) ${ }^{2}$. Advancing with age, the progressive glycosphingolipid accumulation, particularly in vascular endothelial cells, cardiomyocytes and renal podocytes, leads to cerebrovascular disease, cardiomyopathy and renal failure ${ }^{3}$ resulting in severe morbidity and premature death ${ }^{4}$.

In contrast, two other categories of the disease are increasingly diagnosed: the "late-onset" and the "genetic variant of unknown significance" (GVUS) ${ }^{5}$. The former one generally leads to a milder disease in adulthood which often involves a single organ system (usually cardiac or renal), while the latter is associated with unclear and variable pathological consequences or no clinical disease at all ${ }^{6}$.

Data on blood pressure (BP) monitoring in literature are scanty, however, those available have revealed a significant prevalence of hypertension in AFD patients, especially in cases with moderate to severe kidney impairment ${ }^{7-11}$ and it becomes more prevalent with the progression of the renal disease ${ }^{8}$.

Kleinert et al. examined uncontrolled hypertension, defined as systolic BP (SBP) $\geq 130 \mathrm{mmHg}$ and/or diastolic BP (DBP) $\geq 80 \mathrm{mmHg}$, among 391 patients with AFD participating in the Fabry Outcome Survey (FOS). Uncontrolled hypertension was diagnosed in $52.4 \%$ of the participants (57\% males and $47 \%$ females) progressively increasing with the decline of glomerular filtration rate (GFR) estimated using the short Modification of Diet in Renal Disease (MDRD) formula ${ }^{7}$.

On the contrary, analysing 105 male AFD patients, Branton et al. showed that hypertension (SBP $\geq 140 \mathrm{mmHg}$ and/or DBP $\geq 90 \mathrm{mmHg}$ ) was diagnosed in only one-third of the patients (30\%) and, in most cases, was not present until the onset, or long after the onset, of renal impairment or end-stage renal disease (ESRD) ${ }^{12}$.

Given the wide and complex spectrum of AFD and the potential negative impact of hypertension on target organ damage (kidney, heart and nervous system), an appropriate algorithm for the diagnosis and therapy of elevated blood pressure may improve morbidity and mortality of AFD patients ${ }^{13}$. 
Our aim was therefore to analyse BP levels and compare routine BP measurement standards, risk factors related to elevated blood pressure and possible clinical correlations in our AFD patient cohort. Additionally, we performed an analysis of the possible aetiologies of hypertension in these patients.

\section{Methods}

Patients aged 18 years or older with a prior confirmed genetic diagnosis of AFD were eligible for inclusion. Between January 2015 and May 2019 , thirty-two patients referred to the Fabry Disease Unit, Nephrology Division of San Gerardo Hospital (Monza, Italy) were enrolled in the study. They were twenty-four (75\%) females and eight (25\%) males; all of them gave informed consent.

The included patients $(n=32)$ accounted for $80 \%$ of the total eligible Fabry patient cohort $(n=40)$ at our institution in that time-period. Accordingly, the following patients $(n=8)$ were excluded from the study: four patients refused or failed to perform the 24-hours ambulatory blood pressure monitoring (ABPM) and four subjects reported insufficient clinical or echocardiographic data.

Information on patients' demographic characteristics such as sex, race, age, smoke habit, alcohol consumption, glucose intolerance or diabetes, obesity (body mass index $(\mathrm{BMI}) \geq 30$ ), dyslipidaemia, sedentary lifestyle (no physically demanding job and no regular physical activity) and positive family history for hypertension are shown in Table 1.

During the programmed follow-up visits, we evaluated signs and symptoms of AFD, in particular renal (proteinuria, kidney failure) and cardiac (ECG and echocardiographic changes) alterations (Table 2).

\section{Blood pressure measurement}

Data regarding BP values were obtained by ABPM (oscillometric method) and were then paired with those recorded during our clinic evaluation (sitting and standing) and home BP monitoring (morning and evening for at least three times).

Therefore, hypertension was defined according to 2018 European Society of Cardiology/European Society of Hypertension (ESC/ESH) Guidelines for the management of arterial hypertension ${ }^{14}$ (Supplemental Digital Content 1).

\section{Treatment evaluation}

Information on medications such as enzyme replacement therapy (ERT) or oral chaperone therapy and antihypertensive drugs (calcium channel blockers, ACE inhibitors (ACEi), angiotensin II receptor antagonists (ARBs), diuretics and beta-blockers) were reported (Table 1).

\section{Renal function assessment}

The glomerular filtration rate (GFR) was measured along with the albumin-to-creatinine ratio (ACR) and the proteinuria levels on a 24-hours urine collection sample at the time of the ABPM. According to the Kidney Disease Improving Global Outcomes (KDIGO) guidelines, chronic kidney disease was classified in different levels ranging from G1 to G5 on the GFR scale and A1 to A3 on the ACR range ${ }^{15}$ (Supplemental Digital Content 2). Serum electrolyte test and automatic urinary sediment analyses were also performed by our routine biochemistry laboratory.

\section{Echocardiographic examination}

Parasternal and apical 2-dimensional echocardiograms (2D) were acquired according to the American Society of Echocardiography/European Association of Echocardiography (ASE/EAE) recommendations ${ }^{16}$. All echocardiograms were performed and analysed by the same experienced cardiologist. The inter-ventricular septum thickness (IVSd), the posterior wall thickness (PWd) and the diameter of the left ventricle (LVDd) were measured at end-diastole. Left ventricular mass index (LVMi) and relative wall thickness (RWT) were calculated using the adequate equations 17,18. The reference ranges for these values were: RWT $\leq 0.42$ in males and females, $L V M i<115 \mathrm{~g} / \mathrm{m}^{2}$ in males and $<95 \mathrm{~g} / \mathrm{m}^{2}$ in females. The geometric changes of the left ventricle were classified, using the LVMi and RWT values, in concentric hypertrophy (elevated RWT with increased LVMi), concentric remodelling (elevated RWT with normal LVMi), eccentric hypertrophy (normal RWT with increased LVMi) and normal geometry (normal RWT with normal LVMi). Data on the ejection fraction (EF), left atrial volume index (LAVi), tricuspid annular plane systolic excursion (TAPSE), E/e' and E/A ratio were reported.

\section{Fabry Stabilization Index assessment}

The severity and the stability of the organ involvement were assessed through the Fabry Stabilization Index (FASTEX) ${ }^{19}$. We calculated the "raw score" based on seven clinical parameters: pain, cerebrovascular events, proteinuria, glomerular filtration rate, echocardiography parameters, electrocardiographic parameters and New York Heart Association (NYHA) class. Two "raw scores" were compared in two consecutive visits one year apart, obtaining the FASTEX index: a percentage threshold value that indicates if the patient has worsened between one visit and another. When FASTEX is equal to or greater than $20 \%$, the patient is defined not stable, whilst if it is less than $20 \%$ the patient is in stable conditions. The score was obtained using the online FASTEX index tool ${ }^{20}$. 


\section{Statistical analysis}

Data are given as means \pm standard deviation (SD). Statistical analysis was performed using Microsoft Excel and SPSS version 25.0. Mean values were compared by Student's t-test for independent samples and categorical data were analysed by the Fisher's exact test when appropriate.

Table 1

2018 European Society of Cardiology/European Society of Hypertension (ESC/ESH) Guidelines for the management of hypertension.

\begin{tabular}{|c|c|c|c|c|}
\hline Characteristics & $\begin{array}{l}\text { Overall } \\
(n=32)\end{array}$ & $\begin{array}{l}\text { Normotension } \\
(n=22)\end{array}$ & $\begin{array}{l}\text { Hypertension } \\
(n=6)\end{array}$ & $\begin{array}{l}\text { Treated hypertension } \\
(n=4)\end{array}$ \\
\hline Sex: female, n (\%) & $24(75)$ & $16(72.7)$ & $6(100)$ & $2(50)$ \\
\hline Race: Caucasian, n (\%) & $32(100)$ & $22(100)$ & $6(100)$ & $4(100)$ \\
\hline Age (years), mean (SD) & $50.3(12.4)$ & $48.4(12.4)$ & $57.8(10.9)$ & $49.3(12.6)$ \\
\hline \multicolumn{5}{|l|}{ Therapy, n (\%) } \\
\hline Anti-proteinuric therapy & $7(21.9)$ & $7(31.8)$ & $0(0)$ & $0(0)$ \\
\hline ACE-inhibitors & $5(71.4)$ & $5(71.4)$ & $0(0)$ & $0(0)$ \\
\hline Angiotensin II receptor antagonists & $2(28.6)$ & $2(28.6)$ & $0(0)$ & $0(0)$ \\
\hline Anti-hypertensive therapy & $4(12.5)^{\S}$ & $0(0)$ & $0(0)$ & $4(100)^{\S}$ \\
\hline Beta-blockers & $2(50)$ & $0(0)$ & $0(0)$ & $2(50)$ \\
\hline Diuretics & $1(25)$ & $0(0)$ & $0(0)$ & $1(25)$ \\
\hline ACE-inhibitors & $2(50)$ & $0(0)$ & $0(0)$ & $2(50)$ \\
\hline Calcium channel blockers & $1(25)$ & $0(0)$ & $0(0)$ & $1(25)$ \\
\hline Enzyme replacement therapy & $17(53.1)$ & $12(54.5)$ & $3(50)$ & $2(50)$ \\
\hline Agalsidase-alpha & $7(41.2)$ & $5(41.7)$ & $1(33.3)$ & $1(50)$ \\
\hline Agalsidase-beta & $10(58.8)$ & $7(58.3)$ & $2(66.7)$ & $1(50)$ \\
\hline Chaperonic therapy & $2(6.3)$ & $0(0)$ & $1(16.7)$ & $1(25)$ \\
\hline \multicolumn{5}{|l|}{ Risk factors for hypertension, $\mathrm{n}(\%)$} \\
\hline Dyslipidaemia & $14(43.8)$ & $10(45.5)$ & $3(50)$ & $1(25)$ \\
\hline Sedentary life-style & $18(56.3)$ & $10(45.5)$ & $5(83.3)$ & $3(75)$ \\
\hline Obesity & $3(9.4)$ & $2(9.1)$ & $1(16.7)$ & $0(0)$ \\
\hline $\mathrm{BMI}\left(\mathrm{kg} / \mathrm{m}^{2}\right)$, mean $(\mathrm{SD})$ & $23.3(4.1)$ & $23.4(4.2)$ & $22.8(4.6)$ & $23.9(3.2)$ \\
\hline Smoke & $7(21.9)$ & $5(22.7)$ & $1(16.7)$ & $1(25)$ \\
\hline Alcohol consumption & $3(9.4)$ & $2(9.1)$ & $1(16.7)$ & $0(0)$ \\
\hline Positive family history & $12(37.5)$ & $8(36.4)$ & $2(33.3)$ & $2(50)$ \\
\hline Glucose intolerance & $1(3.1)$ & $0(0)$ & $1(16.7)$ & $0(0)$ \\
\hline CKD stage G1-G2 / A1 & $26(81.25)$ & 19 (86.4) & $4(66.7)$ & $3(75)$ \\
\hline CKD stage G3a-G5 / A2-A3 & $6(18.75)$ & $3(13.6)$ & $2(33.3)$ & $1(25)$ \\
\hline \multicolumn{5}{|l|}{ Renal function, mean (SD) } \\
\hline Serum creatinine $(\mathrm{mg} / \mathrm{dl})$ & $0.88(0.2)$ & $0.85(0.2)$ & $0.97(0.2)$ & $0.90(0.1)$ \\
\hline GFR $\left(\mathrm{ml} / \mathrm{min} / 1.73 \mathrm{~m}^{2}\right)$ & $95.6(35.5)$ & $104.15(34.3)$ & $70.38(40.8)$ & $86.43(10.2)$ \\
\hline Proteinuria (mg/24 h) & $135.3(102.9)$ & $136.7(90.8)$ & $121.33(41.7)$ & $148.50(219.2)$ \\
\hline ACR $(\mathrm{mg} / \mathrm{g})$ & $39.4(60.6)$ & $36.0(67.8)$ & $50.78(44,0)$ & $40.60(46.1)$ \\
\hline
\end{tabular}

Characteristics of the patients. 
Table 2

Kidney Disease Improving Global Outcomes (KDIGO) Classification and Prognosis of Chronic Kidney Disease 2012.

\begin{tabular}{|c|c|c|c|c|}
\hline & $\begin{array}{l}\text { Overall, } \\
\text { mean (\%) }\end{array}$ & $\begin{array}{l}\text { Normotension, } \\
\text { mean (\%) }\end{array}$ & $\begin{array}{l}\text { Hypertension, } \\
\text { mean (\%) }\end{array}$ & $\begin{array}{l}\text { Treated hypertension, } \\
\text { mean (\%) }\end{array}$ \\
\hline & $\mathrm{n}=32$ & $\mathrm{n}=\mathbf{2 2}$ & $n=6$ & $\mathrm{n}=4$ \\
\hline Nervous system & $21(65.6)$ & $14(63.6)$ & $6(100)$ & $1(25)$ \\
\hline Neuropathic pain & $15(71.4)$ & $9(64.3)$ & $5(83.3)$ & $1(100)$ \\
\hline Dyshidrosis & $9(42.9)$ & $7(50)$ & $2(33.3)$ & $0(0)$ \\
\hline White matter lesions & $3(14.3)$ & $3(21.4)$ & $0(0)$ & $0(0)$ \\
\hline Neurological events & $1(4.8)$ & $1(7.1)$ & $0(0)$ & $0(0)$ \\
\hline Renal system & $10(32.3)$ & $6(27.3)$ & $3(50)$ & $1(25)$ \\
\hline $\mathrm{ACR}>30 \mathrm{mg} / \mathrm{g}$ & $6(60)$ & $3(50)$ & $2(66.7)$ & $1(100)$ \\
\hline $\mathrm{GFR}<60 \mathrm{ml} / \mathrm{min}$ & $3(30)$ & $1(16.7)$ & $2(66.7)$ & $0(0)$ \\
\hline $\mathrm{GFR}>150 \mathrm{ml} / \mathrm{min}$ & $4(40)$ & $3(50)$ & $1(33.3)$ & $0(0)$ \\
\hline Cardiac system & $29(90.6)$ & $19(86.4)$ & $6(100)$ & $4(100)$ \\
\hline ECG alterations & $23(79.3)$ & $15(78.9)$ & $6(100)$ & $2(50)$ \\
\hline Echo alterations & $20(68.9)$ & $11(57.9)$ & $6(100)$ & $3(75)$ \\
\hline Cardiac events & $0(0)$ & $0(0)$ & $0(0)$ & $0(0)$ \\
\hline Gastrointestinal system & $12(37.5)$ & $8(36.4)$ & $3(50)$ & $1(25)$ \\
\hline Abdominal pain & $10(83.3)$ & $7(87.5)$ & $2(66.7)$ & $1(100)$ \\
\hline Nausea & $2(16.7)$ & $1(12.5)$ & $1(33.3)$ & $0(0)$ \\
\hline Diarreha & $3(25)$ & $2(25)$ & 1 (33.3) & $0(0)$ \\
\hline Cutaneous system & $4(12.5)$ & $4(18.2)$ & $0(0)$ & $0(0)$ \\
\hline Angiokeratomas & $4(100)$ & $4(100)$ & $0(0)$ & $0(0)$ \\
\hline Respiratory system & $7(21.9)$ & $7(31.9)$ & $0(0)$ & $0(0)$ \\
\hline Shortness of breath & $7(100)$ & $7(100)$ & $0(0)$ & $0(0)$ \\
\hline Respiratory failure & $0(0)$ & $0(0)$ & $0(0)$ & $0(0)$ \\
\hline Sensory system & $22(68.8)$ & $15(68.2)$ & $5(83.3)$ & $2(50)$ \\
\hline Cornea verticillata & $15(68.2)$ & $9(60)$ & $4(80)$ & $2(100)$ \\
\hline Hearing impairment & $7(31.8)$ & $5(33.3)$ & $1(20)$ & $1(50)$ \\
\hline Vertigo & $4(18.2)$ & $4(26.7)$ & $0(0)$ & $0(0)$ \\
\hline
\end{tabular}

Fabry organ involvement.

\section{Results}

\section{General characteristics and risk factor analysis of the overall population}

All patients $(n=32)$ were Caucasian with an average age of $50.3 \pm 12.4(22 \div 69)$ years.

They had mostly a sedentary lifestyle $(n=18)$ and less than a half of them was affected by dyslipidaemia $(n=14)$. Obesity was a rare condition ( $n$ $=3$ ) and the average body mass index was within the normal ranges $(B M I=23.3 \pm 4.1)$. Glucose intolerance and diabetes were uncommon $(n=1)$ and only few of them were smokers $(n=7)$ or alcohol consumers $(n=3)($ Table 1$)$.

\section{Treatment evaluation}


Out of the whole population ( $n=32)$, few $(n=4)$ patients had a previously established diagnosis of hypertension and were under anti-hypertensive drugs such as calcium channel blockers $(n=1)$ or ACEi $(n=2)$ in combination with beta-blockers $(n=2)$ or diuretics $(n=1)$. Others $(n=7)$ were taking anti-proteinuric drugs (ACEi and ARBs), but all of them had normal BP levels (office and home monitoring) even before starting the therapy. The remaining $(n=21)$ did not have a known diagnosis of hypertension and were not taking any anti-hypertensive drug.

The $59.4 \%$ ( $n=19,12$ females and 7 males) of the patients were under specific treatment for AFD, two were taking migalastat and the majority ( $\mathrm{n}$ = 17) were on enzyme replacement therapy (ERT): $41.2 \%$ on agalsidase-alpha and $58.8 \%$ on agalsidase-beta.

\section{Genotype and phenotype}

Patients were carriers of heterogeneous GLA gene mutations. The majority $(n=23)$ expressed a classic or likely-classic ${ }^{21}$ mutation while seven had a late-onset one; the most common was the p.Asn215Ser $(n=6)$ which have been reported to be associated with a late-onset cardiac variant 22. Two patients had a mutation not previously described in literature (Table 3).

Accordingly, the phenotypic expression was varied, with more than a half of the patients presenting with multi-organ involvement. The signs and symptoms mainly involved the nervous (neuropathic pain and dyshidrosis), cardiac (ECG and echocardiographic alterations) and renal (proteinuria and renal failure) systems (Table 2). 
Table 3

23

\begin{tabular}{|c|c|c|c|c|c|c|c|c|c|}
\hline Patients & Age & Sex & cDNA change & Protein change & $\begin{array}{l}\text { Coding } \\
\text { effect }\end{array}$ & $\begin{array}{l}\text { Clinical } \\
\text { significance }\end{array}$ & $\begin{array}{l}\text { Cardiac } \\
\text { involvement }\end{array}$ & $\begin{array}{l}\text { Renal } \\
\text { involvement }\end{array}$ & Hypertension \\
\hline 1 & 65 & $\mathrm{~F}$ & c. $515 \mathrm{G}>\mathrm{A}$ & p.Cys172Tyr & Missense & Classic & Yes & No & No \\
\hline 2 & 61 & $\mathrm{~F}$ & c. $155 \mathrm{G}>\mathrm{A}$ & p.Cys52Tyr & Missense & Classic & Yes & No & Treated \\
\hline 3 & 57 & $\mathrm{~F}$ & c. $155 \mathrm{G}>\mathrm{A}$ & p.Cys52Tyr & Missense & Classic & Yes & Yes & No \\
\hline 4 & 51 & $\mathrm{~F}$ & c. $824 \mathrm{~T}>\mathrm{A}$ & p.Leu275His & Missense & Classic & Yes & Yes & No \\
\hline 5 & 56 & $\mathrm{~F}$ & c. $708 \mathrm{G}>\mathrm{C}$ & p.Trp236Cys & Missense & Classic & Yes & No & No \\
\hline 6 & 32 & $\mathrm{~F}$ & c. $658 \mathrm{C}>\mathrm{T}$ & p.Arg220Ter & Nonsense & Classic & Yes & No & No \\
\hline 7 & 63 & $\mathrm{~F}$ & c. $658 \mathrm{C}>\mathrm{T}$ & p.Arg220Ter & Nonsense & Classic & Yes & Yes & Yes \\
\hline 8 & 22 & M & c. $901 \mathrm{C}>\mathrm{T}$ & p.Arg301Ter & Nonsense & Classic & Yes & Yes & No \\
\hline 9 & 45 & $\mathrm{~F}$ & c. $901 \mathrm{C}>\mathrm{T}$ & p.Arg301Ter & Nonsense & Classic & Yes & No & No \\
\hline 10 & 39 & $\mathrm{~F}$ & $c .4 C>T$ & p.GIn2Ter & Nonsense & Classic & Yes & Yes & No \\
\hline 11 & 59 & M & $c .4 C>T$ & p.GIn2Ter & Nonsense & Classic & Yes & No & No \\
\hline 12 & 27 & $M$ & c. $4 \mathrm{C}>\mathrm{T}$ & p.GIn2Ter & Nonsense & Classic & Yes & Yes & No \\
\hline 13 & 39 & M & c. $1024 \mathrm{C}>\mathrm{T}$ & p.R342X & Nonsense & Classic & No & No & No \\
\hline 14 & 63 & $\mathrm{~F}$ & c. $233 \mathrm{C}>\mathrm{G}$ & p.Ser78Ter & Nonsense & Classic & No & No & No \\
\hline 15 & 60 & M & c. $644 A>G$ & p.Asn215Ser & Missense & Later Onset & Yes & No & No \\
\hline 16 & 46 & $\mathrm{~F}$ & c. $644 \mathrm{~A}>\mathrm{G}$ & p.Asn215Ser & Missense & Later Onset & Yes & No & No \\
\hline 17 & 56 & $\mathrm{~F}$ & c. $644 \mathrm{~A}>\mathrm{G}$ & p.Asn215Ser & Missense & Later Onset & Yes & No & No \\
\hline 18 & 61 & $\mathrm{~F}$ & c. $644 \mathrm{~A}>\mathrm{G}$ & p.Asn215Ser & Missense & Later Onset & No & No & No \\
\hline 19 & 34 & M & c. $644 \mathrm{~A}>\mathrm{G}$ & p.Asn215Ser & Missense & Later Onset & Yes & No & Treated \\
\hline 20 & 49 & $\mathrm{~F}$ & c. $644 A>G$ & p.Asn215Ser & Missense & Later Onset & Yes & Yes & Yes \\
\hline 21 & 39 & $\mathrm{~F}$ & c. $272 \mathrm{~T}>\mathrm{C}$ & p.lle91Thr & Missense & Later Onset & Yes & No & No \\
\hline 22 & 49 & M & c. $370-2 A>G$ & p.Y123C & $\begin{array}{l}\text { Consensus } \\
\text { splice }\end{array}$ & $\begin{array}{l}\text { Likely } \\
\text { Classic }\end{array}$ & Yes & Yes & No \\
\hline 23 & 69 & $\mathrm{~F}$ & c.428dupC & p.G144Qfs*12 & Frameshift & $\begin{array}{l}\text { Likely } \\
\text { Classic }\end{array}$ & Yes & Yes & Yes \\
\hline 24 & 57 & $\mathrm{~F}$ & c. $806 \mathrm{G}>\mathrm{U}$ & p.G261C & Missense & $\begin{array}{l}\text { Likely } \\
\text { Classic }\end{array}$ & Yes & No & No \\
\hline 25 & 58 & $M$ & c. $806 \mathrm{G}>\mathrm{U}$ & p.G261C & Missense & $\begin{array}{l}\text { Likely } \\
\text { Classic }\end{array}$ & Yes & Yes & Treated \\
\hline 26 & 54 & $\mathrm{~F}$ & c. $806 \mathrm{G}>\mathrm{U}$ & p.G261C & Missense & $\begin{array}{l}\text { Likely } \\
\text { Classic }\end{array}$ & Yes & Yes & Yes \\
\hline 27 & 44 & $\mathrm{~F}$ & c. $824 \mathrm{~T}>\mathrm{A}$ & p.Leu275His & Missense & $\begin{array}{l}\text { Likely } \\
\text { Classic }\end{array}$ & Yes & No & Treated \\
\hline 28 & 40 & $\mathrm{~F}$ & $\begin{array}{l}\text { c. } 621 \mathrm{~T}>\mathrm{A} \\
(\mathrm{c} .427 \mathrm{G}>\mathrm{A})\end{array}$ & $\begin{array}{l}\text { p.Y207X } \\
\text { (p.A143T) }\end{array}$ & $\begin{array}{l}\text { Nonsense } \\
\text { (Missense) }\end{array}$ & $\begin{array}{l}\text { Likely } \\
\text { Classic } \\
\text { (Benign) }\end{array}$ & Yes & No & No \\
\hline 29 & 69 & $\mathrm{~F}$ & $\begin{array}{l}\text { c. } 621 \mathrm{~T}>\mathrm{A} \\
(\mathrm{c} .427 \mathrm{G}>\mathrm{A})\end{array}$ & $\begin{array}{l}\text { p.Y207X } \\
\text { (p.A143T) }\end{array}$ & $\begin{array}{l}\text { Nonsense } \\
\text { (Missense) }\end{array}$ & $\begin{array}{l}\text { Likely } \\
\text { classic } \\
\text { (Benign) }\end{array}$ & Yes & No & Yes \\
\hline 30 & 63 & $\mathrm{~F}$ & c. 124-125delAT & p.Met42GlyfsTer13 & Deletion & Classic & Yes & No & No \\
\hline 31 & 43 & $\mathrm{~F}$ & $\begin{array}{l}\text { c.634- } \\
\text { 639delAAAAG }\end{array}$ & p.Q212fsX230 & UND & UND & Yes & No & Yes \\
\hline 32 & 39 & $\mathrm{~F}$ & c.73delGfsX120 & p.Asp25Thrfs96x & UND & UND & Yes & No & No \\
\hline
\end{tabular}




\section{Renal function assessment}

Chronic kidney disease ranged mainly from G1/A1 to G2/A1 $(n=26)$ while few patients $(n=6)$ presented a more advanced renal failure with Chronic kidney disease (CKD) stages from G3a to G5 and/or albuminuria stages from A2 to A3 (Table 1). They were three males and three females, five carrying a classical mutation and one a late-onset one.

\section{Blood pressure measurement}

With the 24-hours ABPM we diagnosed elevated BP in $18.75 \%(n=6)$ of the population while the $81.25 \%(n=26)$ was normotensive, including those $(n=4)$ with a prior diagnosis of hypertension and under adequate anti-hypertensive therapy.

\section{Patients with newly detected hypertension $(n=6)$ \\ 24-hours ambulatory blood pressure monitoring (ABPM)}

In the six patients with uncontrolled hypertension, ABPM showed a mean SBP of $138.17 \pm 4.88 \mathrm{mmHg}$ and a mean DBP of $81.17 \pm 3.66 \mathrm{mmHg}$; half of them presented a non-dipper pattern. They were all females, four carrying a classic or likely-classic mutation, one a late-onset and another a not-previously-described mutation.

\section{Office and home blood pressure monitoring}

One-half of the uncontrolled hypertensive patients based on ABPM $(n=3)$ showed normal office blood pressure measurements (SBP $130 \pm$ $2.6 \mathrm{mmHg}$, DBP $82.33 \pm 2.5 \mathrm{mmHg}$ ) and reported normal home values (SBP $121 \pm 8.9 \mathrm{mmHg}$, DBP $82.33 \pm 2.1 \mathrm{mmHg}$ ). The other half of these subjects $(n=3)$ presented also elevated home and office blood pressure.

The results of the home monitoring revealed a mean SBP of $133.33 \pm 14.77 \mathrm{mmHg}$ and a mean DBP of $89.17 \pm 7.63 \mathrm{mmHg}$, while the office measurements presented a mean SBP of $144.17 \pm 16.07 \mathrm{mmHg}$ and a DBP of $90.83 \pm 9.91 \mathrm{mmHg}$, without differences between sitting and standing (Table 4).

\section{General characteristics of the hypertensive population}

The six hypertensive women had an average age of $58 \pm 10.9(43 \div 69)$ years old, but they were not significatively older $(p>0.05)$ compared with the normotensive patients which had an average age of $48.4 \pm 12.4(22 \div 69)$ years.

\section{Risk factors analysis}

Hypertensive patients had mostly a sedentary life-style $(n=5,83.3 \%)$, half of them $(n=3)$ had a known diagnosis of dyslipidaemia and one was considered obese with a BMI $\otimes 30$. Smoking habit and alcohol consumption were a rare condition $(n=1)$. They presented a lower $(p<0.05)$ glomerular filtration rate compared with the normotensive patients (Table 1).

\section{Phenotype and genotype}

All the hypertensive patients had cardiological involvement (electrocardiogram and/or echocardiogram alterations) and peripheral neurological manifestations with acroparesthesias and/or hypohidrosis. Three patients (50\%) presented with gastrointestinal symptoms, two out of three experiencing recurrent episodes of abdominal pain and one complaining of frequent nausea and diarreha. No one had pulmonary or cutaneous involvement. Three patients (50\%) presented with renal impairment, two with CKD stage G3a/A2 and the patient with BMI > 30 showed hyperfiltration (GFR >150 ml/min) (Table 2). No electrolytic imbalance or urinary sediment alterations were detected.

No statistically significant correlations have been shown between the type of mutation, classical or late-onset, and the development of hypertension $(p>0.05)$.

\section{Patients with normal blood pressure $(n=22)$}

\section{Ambulatory, office and home blood pressure monitoring}

In the normotensive group, the ABPM revealed a mean SBP of $111.64 \pm 10.61 \mathrm{mmHg}$ and a mean DBP of $65.68 \pm 6.30 \mathrm{mmHg}$.

The results of the office monitoring showed a SBP of $119.09 \pm 11.48 \mathrm{mmHg}$ and a DBP of $73.64 \pm 8.47 \mathrm{mmHg}$ while the home measurements a SBP of $114.32 \pm 10.20 \mathrm{mmHg}$ and a DBP of $65.91 \pm 6.40 \mathrm{mmHg}$. Only one (3.8\%) patient presented with elevated BP values at the office monitoring, probably due to the "white coat syndrome" (Table 4).

General characteristics and risk factors analysis of the normotensive population 
Almost half of the normotensive patients had a sedentary life-style and a known diagnosis of dyslipidaemia. Two were considered obese. Smoking habit and alcohol consumption were a rare condition (respectively $n=5$ and $n=2)($ Table 1 ).

\section{Genotype and phenotype}

Nearly all had cardiological $(n=19,86.4 \%)$ involvement, and more than a half of them had sensorial $(n=15)$, neurological $(n=14)$ and renal $(n=6)$ involvement (Table 2). In this group sixteen (72.7\%) patients had a classic or "likely classic" mutation, five a late-onset and one a not-previouslydescribed one (Table 5).

\section{Patients with treated hypertension $(n=4)$}

The four patients with a previously known diagnosis of hypertension and currently on antihypertensive therapy were analysed separately from the other patients (Table 1).

\section{Ambulatory, office and home blood pressure monitoring}

This group presented a pressure profile (ABPM, home and outpatient monitoring) well controlled by the current anti-hypertensive therapy. At the ABPM they showed a normal mean SBP of $116.75 \pm 1.26 \mathrm{mmHg}$ and a mean DBP of $72.5 \pm 3.70 \mathrm{mmHg}$ along with normal office and home BP monitoring (Table 4).

\section{Genotype and phenotype}

They mostly expressed a classical phenotype $(n=3,75 \%)$ (Table 3) with mainly cardiac involvement $(n=3,75 \%)($ Table 2$)$.

Table 4

\begin{tabular}{|c|c|c|c|c|c|c|c|}
\hline Type of measure & $\begin{array}{l}\text { Values } \\
(\mathrm{mmHg})\end{array}$ & $\begin{array}{l}\text { Overall, } \\
\text { mean (SD) }\end{array}$ & $\begin{array}{l}\text { Normotension, } \\
\text { mean (SD) }\end{array}$ & $\begin{array}{l}\text { Hypertension, } \\
\text { mean (SD) }\end{array}$ & p-value & $\begin{array}{l}\text { Treated hypertension, } \\
\text { mean (SD) }\end{array}$ & p-value \\
\hline \multirow{2}{*}{$\begin{array}{l}\text { Ambulatory BP } \\
\text { (Daytime) }\end{array}$} & Systolic & $118.22(11.78)$ & $114.41(10.91)$ & $132.00(7.38)$ & $<0.001$ & $118.5(4.73)$ & 0.245 \\
\hline & Diastolic & 72.09 (8.99) & $68.82(7.10)$ & $83.83(5.71)$ & $<0.001$ & $72.5(8.54)$ & 0.465 \\
\hline \multirow{2}{*}{$\begin{array}{l}\text { Ambulatory BP } \\
\text { (Night-time) }\end{array}$} & Systolic & $106.00(13.32)$ & $102.64(12.96)$ & $117.17(13.3)$ & 0.045 & $107.75(4.99)$ & 0.194 \\
\hline & Diastolic & $63.13(7.77)$ & $60.14(4.75)$ & $73.33(9.00)$ & 0.014 & $64.25(7.41)$ & 0.353 \\
\hline \multirow{2}{*}{$\begin{array}{l}\text { Ambulatory BP } \\
(24 \mathrm{~h})\end{array}$} & Systolic & $117.25(13.69)$ & $111.64(10.61)$ & $138.17(4.88)$ & $<0.001$ & $116.75(1.26)$ & 0.040 \\
\hline & Diastolic & $69.44(8.26)$ & $65.68(6.30)$ & $81.17(3.66)$ & $<0.001$ & $72.50(3.70)$ & 0.021 \\
\hline \multirow[t]{2}{*}{ Office BP } & Systolic & $124.38(15.20)$ & $119.09(11.48)$ & $144.17(16.07)$ & 0.010 & $123.75(6.85)$ & 0.307 \\
\hline & Diastolic & $76.72(10.92)$ & $73.64(8.47)$ & $90.83(9.91)$ & 0.006 & $72.5(8.66)$ & 0.820 \\
\hline \multirow[t]{2}{*}{ Home BP } & Systolic & $118.91(13.12)$ & $114.32(10.20)$ & $133.33(14.77)$ & 0.023 & $122.50(9.95)$ & 0.202 \\
\hline & Diastolic & $70.94(11.06)$ & $65.91(6.40)$ & $89.17(7.63)$ & $<0.001$ & $71.25(5.56)$ & 0.151 \\
\hline
\end{tabular}

Blood pressure monitoring.

\section{Echocardiographic examination}

The echocardiogram examination showed an all-over high prevalence of cardiac hypertrophy; thirteen (40.6\%) patients presented a concentric hypertrophy, seven $(21.9 \%)$ a concentric remodelling and twelve $(37.5 \%)$ had normal parameters. All the untreated hypertensive patients $(n=6)$ were diagnosed with concentric hypertrophy with a significantly higher LVPWD $(p<0.05)$ and LVMi $(p<0.05)$ compared to the normotensive patients. All the other echocardiographic parameters were not significantly different between hypertensive and normotensive patients.

All the treated hypertensive patients $(n=4)$ presented a concentric hypertrophy, but no statistically significant differences were found between this group and the normotensive one (Table 5). 
Table 5

\begin{tabular}{|c|c|c|c|c|c|c|}
\hline & $\begin{array}{l}\text { Overall, } \\
\text { mean (SD) }\end{array}$ & $\begin{array}{l}\text { Normotension, } \\
\text { mean (SD) }\end{array}$ & $\begin{array}{l}\text { Hypertension, } \\
\text { mean (SD) }\end{array}$ & p-value & $\begin{array}{l}\text { Treated Hypertension } \\
\text { mean (SD) }\end{array}$ & p-value \\
\hline $\operatorname{LVDd}(\mathrm{cm})$ & $4.35(0.38)$ & $4.35(0.43)$ & $4.35(0.20)$ & 0.485 & $4.35(0.26)$ & 0.489 \\
\hline PWd (cm) & $1.04(0.20)$ & $0.99(0.18)$ & $1.18(0.22)$ & 0.044 & $1.11(0.14)$ & 0.101 \\
\hline IVSd (cm) & $1.13(0.26)$ & $1.05(0.22)$ & $1.32(0.33)$ & 0.055 & $1.28(0.22)$ & 0.066 \\
\hline RWT & $0.48(0.10)$ & $0.46(0.10)$ & $0.55(0.11)$ & 0.118 & $0.51(0.09)$ & 0.346 \\
\hline LVMi $\left(g / m^{2}\right)$ & $97.44(29.03)$ & $90.37(28.13)$ & $115.82(25.26)$ & 0.033 & $108.75(29.88)$ & 0.162 \\
\hline $\mathrm{EF}(\%)$ & $67.63(4.92)$ & $67.86(4.36)$ & $66.67(5.57)$ & 0.321 & $67.75(7.93)$ & 0.490 \\
\hline$E / e^{\prime}$ & $9.48(2.63)$ & $9.13(3.07)$ & $10.34(0.98)$ & 0.063 & $10.10(0.96)$ & 0.124 \\
\hline LAVi $\left(\mathrm{ml} / \mathrm{m}^{2}\right)$ & $33.66(17.20)$ & $30.77(6.96)$ & $45.67(37.51)$ & 0.188 & $31.50(4.20)$ & 0.393 \\
\hline TAPSE (cm) & $2.28(0.34)$ & $2.33(0.25)$ & $2.15(0.51)$ & 0.221 & $2.25(0.51)$ & 0.392 \\
\hline $\mathrm{E} / \mathrm{A}$ & $1.42(0.56)$ & $1.44(0.57)$ & $1.41(0.66)$ & 0.467 & $1.34(0.46)$ & 0.356 \\
\hline
\end{tabular}

Echocardiographic data

\section{FASTEX assessment}

In the overall study population $(n=32)$, eight $(25 \%)$ patients presented a progressive form of AFD accordingly to the FASTEX score. Four out of the six $(66.7 \%)$ hypertensive subjects were classified as progressive, while a great portion $(86.4 \%)$ of the normotensive group was not. The majority (75\%) of well treated hypertensive patients also showed a stable FASTEX score (Fig. 1).

\section{Discussion}

In this study, the majority of AFD patients ( $\mathrm{n}=22,68.75 \%)$ were normotensive without specific blood pressure control therapy. Four (12.5\%) patients previously diagnosed with hypertension and currently under anti-hypertensive treatment showed a well-controlled blood pressure, while six (18.75\%) were diagnosed for the first time, due to the ABPM measurements, with uncontrolled hypertension. Half of them presented a nondipping blood pressure pattern, which is shown to be associated with additional cardiovascular alterations and organ damage ${ }^{24}$. Of note, half of these patients had normal blood pressure recordings in office and home measurements; masked hypertension was diagnosed with 24 -hours blood pressure measurements highlighting the need of standardized long-term recordings, especially in patients with concomitant early signs of cardiomyopathy and/or nephropathy.

Our findings in the AFD population are in line with a recent presentation that shows that masked hypertension may have an average prevalence of about $40 \%$ in CKD-patients ${ }^{25}$.

Apart from dyslipidaemia and sedentary lifestyle, the overall population revealed a low prevalence of well-known risk factors for the development of hypertension such obesity, glucose intolerance and diabetes, smoke habit and alcohol consumption.

The hypertensive group showed a higher prevalence of kidney involvement with the development of proteinuria and renal failure, supporting previous reports ${ }^{7-11}$. Almost all of them presented electrocardiographic and echocardiographic alterations and the echocardiographic analysis highlighted that patients with uncontrolled hypertension showed a worse cardiac hypertrophy as compared to the well treated-hypertensive patients.

The majority of the hypertensive patients presented an unstable disease according to the FASTEX analysis, with progressive neurological, cardiac and renal involvement presumably connected with the development of high blood pressure. Moreover, untreated high blood pressure values represent an established risk factor for adverse cardiovascular and kidney outcomes 25,26 .

In addition, hypertensive AFD patients showed a higher prevalence of classical mutations, related to a more severe phenotype compared with the late-onset ones, mainly involving one organ system such as the neuro-vegetative, cardiological or renal one ${ }^{27}$.

In our study, the prevalence of hypertensive patients was lower as compared to several previous published papers ${ }^{7-10}$. For instance, our results are in contrast to the report by Kleinert et al. ${ }^{7}$ which showed a high overall rate of uncontrolled hypertension (57\% in male patients) in a population of 391 patients affected by AFD. We described a lower prevalence of hypertension despite the average age of our patients being higher. Furthermore, the majority of our patients were female, and none of our patients had severe kidney failure, ESRD, needed dialysis or had received a 
kidney transplantation. In addition, the criteria for the diagnosis of hypertension differs slightly between the two studies. It is significant to point out that we analysed a smaller sample size, but our blood pressure monitoring was strictly structured.

Notably, our hypertensive patients were identified by automated and home blood pressure analyses, highlighting the need of well standardized blood pressure measuring routines in the follow-up of Fabry patients as half of them presented with normal office blood pressure. An important observation in our study is the association between non-controlled hypertension and clinically progressive disease, not seen in those patients where blood pressure was well controlled by antihypertensive agents at the time of inclusion. Of note, our observation also highlights the necessity of diagnosing the often-overlooked masked hypertension in AFD patients with early progressive cardiac or renal disease.

Moreover, a study on 10,051 individuals ( $53.2 \%$ female, age $56.2 \pm 16.8$ years) revealed that the prevalence of hypertension in Italy varies from $55-$ $59 \%{ }^{28}$. Taking into account the limitations of a comparison between these studies, our data indicate that hypertension is not a frequent finding in stable and well-monitored AFD patients, who are likely to have a lower prevalence compared to the general population with the same age range. This is consistent with the general concept that Fabry disease often is accompanied by lower blood pressure levels, which in fact may limit the anti-proteinuric use of ACEi/RAS-blockade in many patients ${ }^{29}$.

The mechanisms connecting Fabry disease to the development of hypertension have not been fully studied yet. Hypertension might be a consequence of an AFD associated vascular disease.

It is not known whether the vascular disease originates from the deposition of glycosphingolipids in endothelial cells or in smooth muscle cells in the arterial media layer ${ }^{30,31}$. Growing evidence indicates that the deposition of $\mathrm{Gb} 3$ in the endothelium activates oxidative enzymes such as nicotinamide adenine dinucleotide phosphate (NADPH) oxidases leading to the production of reactive oxygen species (ROS) ${ }^{32}$. Moreover, storage of glycosphingolipids in smooth muscle cells may promote cell proliferation with fibrotic remodelling of the arterial wall leading to arterial wall stiffness resulting in shear stress that may increase the expression of angiotensin 1 and 2 receptors in endothelial cells, increasing ROS, NF-kB, beta-integrin and cyclooxygenase 1 and 2 activity and decreasing nitric oxide synthesis ${ }^{33}$. These mechanisms may lead to oxidative stress and inflammatory damage which promotes endothelial and vascular dysfunction leading to the development of hypertension ${ }^{34}$.

Patients affected by Fabry disease may also develop hypertension due to a direct renal damage ${ }^{8}$ caused by the accumulation of glycolipids within the tubular epithelial cells and the podocytes, leading to tubular atrophy, interstitial fibrosis and segmental and global glomerulosclerosis ${ }^{35}$.

It has long been accepted the concept that renal impairment and hypertension are closely associated ${ }^{36}$. Sustained and untreated high blood pressure values hasten the progression of kidney disease and, in turn, renal damage is clearly related to the development of hypertension ${ }^{37}$.

Another theory speculates that hypertension may be a process unrelated to Fabry disease, such as in the case of essential hypertension ${ }^{12}$. Additionally, kidney biopsy reports have shown concomitant renal diseases causing hypertension that may co-exist with Fabry disease (e.g. focal and segmental glomerular sclerosis, membranous or IgA nephropathy) ${ }^{38-41}$.

However, we can speculate that glycolipids accumulation may also affect the autonomic nervous system causing an impairment of the arterial baroreflex function ${ }^{42}$, leading to an altered blood pressure regulation, with possible orthostatic hypotension and predisposition to syncope ${ }^{9}$ even if we did not diagnosed any orthostatic hypotension in our patients.

The purpose of a close blood pressure control in AFD population is important to reduce cardiovascular events and progressive deterioration of renal function.

We recommend the inclusion of ABPM for a reliable monitoring of BP in routine follow-up of AFD patients. Other studies in CKD patients confirm that the routine office BP measurements provide imprecise reflection of the actual BP load ${ }^{25,43}$ as recommended by the ESC-ESH Guidelines 2018 44

The limitations of our study are the small sample size of the population analysed and the higher prevalence of women compared to men. It is important to underline also the lack in the analysis of the patients eating habits, the sodium intake and natriuresis. Enlisting a control group of non-AFD patients would have helped to better develop the analysis.

The strength of the study are the accurate characterization of the patients and the meticulous BP monitoring according to current guidelines.

\section{Conclusions}

The results of our study underline that hypertension can be found in a restricted portion of stable and well-monitored AFD patients with minimal signs of organ complications, becoming more prevalent in clinically progressive cases. The use of ABPM is of paramount importance to reveal the cases of masked hypertension which, if not properly diagnosed, can contribute to the progressive worsening of the organ damage. We recommend 
a standardised ambulatory long-term blood pressure monitoring program and timely antihypertensive intervention to improve the outcome of AFD patients.

\section{Abbreviations}

\section{ABPM}

ambulatory blood pressure monitoring; ACR:albumin-to-creatinine ratio; DBP:diastolic blood pressure; EF:ejection fraction; ERT:enzyme replacement therapy; FASTEX:Fabry Stabilization Index; FOS:Fabry Outcome Survey; GVUS:genetic variant of unknown significance; IVSd:interventricular septum thickness at end diastole; LAVi:left atrial volume index; LVDd:diameter of the left ventricle at end diastole; LVMi:left ventricular mass index; NADPH:nicotinamide adenine dinucleotide phosphate; PWd:posterior wall thickness at end diastole; Ros:reactive oxygen species; RWT:relative wall thickness; SBP:systolic blood pressure; TAPSE:tricuspid annular plane systolic excursion.

\section{Declarations}

\section{Ethics approval and consent to participate}

The research was conducted in accordance with the World Medical Association Declaration of Helsinki and the institute's Ethics Committee (ComitatoEticoBrianza) approved it (DREGC07006/AGAL19211).

Informed consent was obtained from all participants included in the study.

\section{Consent for publication}

Not applicable.

\section{Availability of data and materials}

The dataset supporting the conclusions of this article is included within the article as additional file.

\section{Competing interests}

E.S. reports receiving a grant from Sanofi Genzyme; positions on the advisory boards of Amicus and Sanofi Genzyme; speaker fees and travel support from Amicus, Sanofi Genzyme, and Shire-Takeda. H.P.M. reports receiving grants from Alexion, Amicus, Sanofi Genzyme, and ShireTakeda. F.P. reports participations at advisory boards, speaker fees and travel support from Amicus, Sanofi Genzyme, and Shire-Takeda. F.R., H.E., A.B, L.R. and S.A. declare no conflict of interest.

\section{Funding}

The authors received no financial support for research, authorship and/or publication of this article.

\section{Authors' contributions}

F.R. conceived the research design, collected patients' data, performed the statistical analysis and wrote the manuscript first draft. H.P.M., E.S. and H.E. actively participated in the writing of the manuscript. F.P. performed the echocardiographic examination. A.B., L.R. and S.A collected patients' data during the clinical visits and implemented the database. H.P.M., E.S. and F.P. provided supervision and mentorship of F.R. during the drafting. All authors were involved in approving the final version of the manuscript.

\section{Acknowledgments}

Our special thanks go to members of the Renal Research Group (Haukeland University Hospital, University of Bergen, Norway), members of the Fabry Group (San Gerardo Hospital, University of Milano-Bicocca, Italy) for their support.

\section{References}

1. Aerts JM, et al Elevated globotriaosylsphingosine is a hallmark of Fabry disease. Proc. Natl. Acad. Sci. U. S. A. 105, 2812-2817 (2008).

2. Germain DP. Fabry disease. Orphanet J Rare Dis. 2010;5:30.

3. Nowak A, Mechtler T, Kasper DC, Desnick RJ. Correlation of Lyso-Gb3 levels in dried blood spots and sera from patients with classic and LaterOnset Fabry disease. Mol Genet Metab. 2017;121:320-4.

4. Hoffmann B. Fabry disease: recent advances in pathology, diagnosis, treatment and monitoring. Orphanet J Rare Dis. $2009 ; 4: 21$. 
5. Tol L, van der, et al. A systematic review on screening for Fabry disease: prevalence of individuals with genetic variants of unknown significance. J Med Genet. 2014;51:1-9.

6. Svarstad E, Marti HP. The Changing Landscape of Fabry Disease. Clin J Am Soc Nephrol. 2020. doi:10.2215/CJN.09480819.

7. Kleinert J, et al. Prevalence of uncontrolled hypertension in patients with Fabry disease. Am J Hypertens. 2006;19:782-7.

8. Ortiz A, et al. Nephropathy in males and females with Fabry disease: cross-sectional description of patients before treatment with enzyme replacement therapy. Nephrol Dial Transplant. 2008;23:1600-7.

9. Schiffmann R, et al. Fabry disease: progression of nephropathy, and prevalence of cardiac and cerebrovascular events before enzyme replacement therapy. Nephrol Dial Transplant. 2009;24:2102-11.

10. Jain G, Warnock DG. Blood pressure, proteinuria and nephropathy in Fabry disease. Nephron Clin Pract. 2011;118:c43-8.

11. Terryn W, et al. Prevalence of Fabry disease in a predominantly hypertensive population with left ventricular hypertrophy. Int $\mathrm{J}$ Cardiol. 2013;167:2555-60.

12. Branton $\mathrm{MH}$, et al. Natural history of Fabry renal disease: influence of alpha-galactosidase A activity and genetic mutations on clinical course. Medicine. 2002;81:122-38.

13. Sunderplassmann G. Uncontrolled hypertension in Fabry disease. Am J Hypertens. 2004;17:206.

14. Williams B, et al. 2018 Practice Guidelines for the management of arterial hypertension of the European Society of Cardiology and the European Society of Hypertension. J Hypertens. 2018;36:2284-309.

15. Eknoyan G, et al. KDIGO 2012 clinical practice guideline for the evaluation and management of chronic kidney disease. Kidney Int. 2013;3:514.

16. Lang RM, et al. Recommendations for Chamber Quantification: A Report from the American Society of Echocardiography's Guidelines and Standards Committee and the Chamber Quantification Writing Group, Developed in Conjunction with the European Association of Echocardiography, a Branch of the European Society of Cardiology. J Am Soc Echocardiogr. 2005;18:1440-63.

17. Devereux RB. Evaluation of cardiac function and vascular structure and function by echocardiography and other noninvasive techniques. Hypertension: Pathophysiology, Ddiagnosis, and Management 1969-1985 (1995).

18. Clarkson PB, Prasad N, MacLeod C, Burchell B, MacDonald TM. Influence of the angiotensin converting enzyme I/D gene polymorphisms on left ventricular diastolic filling in patients with essential hypertension. J Hypertens. 1997;15:995-1000.

19. Mignani R, et al. FAbry STabilization indEX (FASTEX): an innovative tool for the assessment of clinical stabilization in Fabry disease. Clinical Kidney Journal. 2016;9:739-47.

20. Fastex 2.0 Home page. Accessed. January 2020. https://www.fastex.online/Public/publicpage2.php?d6494edqh554b7qvcgfpb3sgb2.

21. Desnick RJ, Chen R, Srinivasan R, Doheny DO, Bishop D. The Fabry disease genotype-phenotype database (dbFGP): an international expert consortium. Mol Genet Metab. 2017;120:41-2.

22. Germain DP, et al. Phenotypic characteristics of the p.Asn215Ser (p.N215S) GLA mutation in male and female patients with Fabry disease: A multicenter Fabry Registry study. Molecular Genetics Genomic Medicine. 2018;6:492-503.

23. Mutation Search. Accessed. January 2020. http://www.dbfgp.org/dbFgp/fabry/Mutation.html.

24. Mezue K, et al. Nocturnal Non-dipping Blood Pressure Profile in Black Normotensives Is Associated with Cardiac Target Organ Damage. Ethn Dis. 2016;26:279-84.

25. Georgianos PI, Agarwal R, Ambulatory BP. Phenotypes and Their Association with Target Organ Damage and Clinical Outcomes in CKD. Clinical journal of the American Society of Nephrology: CJASN. 2020;15:441-3.

26. Schmieder RE. End organ damage in hypertension. Dtsch Arztebl Int. 2010;107:866-73.

27. Azevedo 0, et al. Natural history of the late-onset phenotype of Fabry disease due to the p.F113L mutation. Mol Genet Metab Rep. 2020;22:100565.

28. Tocci G, et al. Trends in Prevalence, Awareness, Treatment, and Control of Blood Pressure Recorded From 2004 to 2014 During World Hypertension Day in Italy. J Clin Hypertens. 2016;18:551-6.

29. Warnock DG. Fabry disease: diagnosis and management, with emphasis on the renal manifestations. Curr Opin Nephrol Hypertens. 2005;14:87-95.

30. Rombach SM, et al. Vasculopathy in patients with Fabry disease: Current controversies and research directions. Mol Genet Metab. 2010;99:99-108.

31. DeGraba T, et al. Profile of endothelial and leukocyte activation in Fabry patients. Ann Neurol. 2000;47:229-33.

32. Napoli C, de Nigris F, Palinski W. Multiple role of reactive oxygen species in the arterial wall. J Cell Biochem. 2001;82:674-82.

33. Rozenfeld P, Feriozzi S. Contribution of inflammatory pathways to Fabry disease pathogenesis. Mol Genet Metab. 2017;122:19-27.

34. Baradaran A, Nasri H, Rafieian-Kopaei M. Oxidative stress and hypertension: Possibility of hypertension therapy with antioxidants. J Res Med Sci. 2014;19:358-67. 
35. Alroy J, Sabnis S, Kopp JB. Renal pathology in Fabry disease. J Am Soc Nephrol. 2002;13(Suppl 2):134-8.

36. Judd E, Calhoun DA. Management of hypertension in CKD: beyond the guidelines. Adv Chronic Kidney Dis. 2015;22:116-22.

37. Santos PCJL, Krieger JE, Pereira AC. Renin-Angiotensin System, Hypertension, and Chronic Kidney Disease: Pharmacogenetic Implications. J Pharmacol Sci. 2012;120:77-88.

38. Svarstad E, et al. Focal and segmental glomerular sclerosis (FSGS) in a man and a woman with Fabry's disease. Clin Nephrol. 2005;63:394401.

39. Zhou W, Ni Z, Zhang M. Hemizygous Fabry disease associated with membranous nephropathy: A rare case report. Clin Nephrol. 2018;90:22731.

40. Maixnerová D, et al. The coincidence of IgA nephropathy and Fabry disease. BMC Nephrol. 2013;14:6.

41. Ren $\mathrm{H}$, et al. Fabry disease and immunoglobulin A nephropathy presenting with Alport syndrome-like findings: A case report. Medicine. 2019;98:e16256.

42. Hilz MJ. Autonomic dysfunction in Fabry disease. Clin Ther. 2010;32:93.

43. Mwasongwe SE, et al. Ambulatory Blood Pressure Phenotypes in Adults Taking Antihypertensive Medication with and without CKD. Clin J Am Soc Nephrol. 2020;15:501-10.

44. Williams B, et al. 2018 ESC/ESH Guidelines for the management of arterial hypertension. Eur Heart J. 2018;39:3021-104.

\section{Figures}

\section{Figure 1}

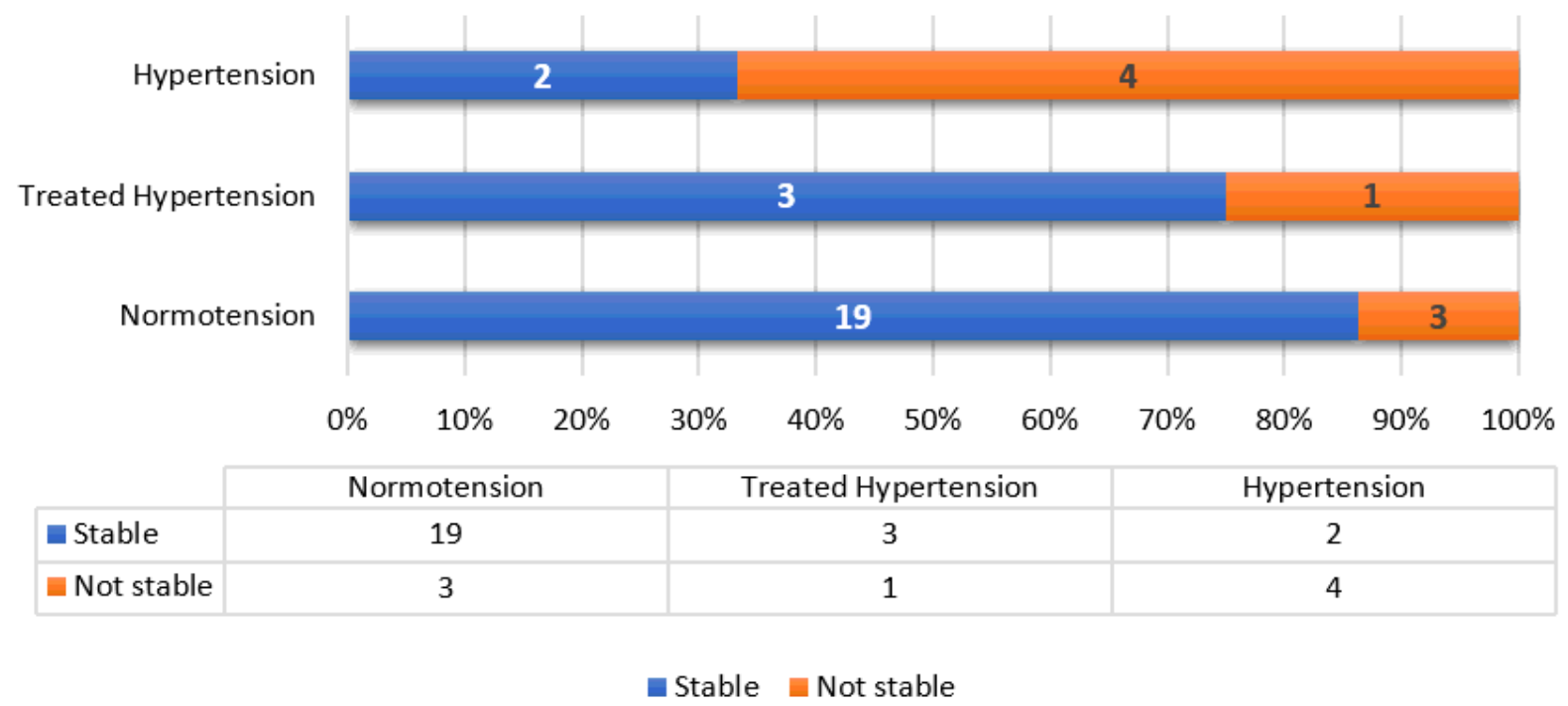

\section{Figure 1}

Fabry Stabilization Index (FASTEX) score.

\section{Supplementary Files}

This is a list of supplementary files associated with this preprint. Click to download.

- Spreadsheet.xlsx

- SupplementalDigitalContent2.pdf

- SupplementalDigitalContent1.pdf 